Research Article

\title{
On the Effect of Different Grips of Handsets on Data Rate in the Measured Channels
}

\author{
Maryam Rahimi and Ehsan Foroozanfard \\ Deptartment of Electronics Systems, Faculty of Engineering and Science, Aalborg University, \\ Fredrik Bajers Vej 7, 9220 Aalborg, Denmark \\ Correspondence should be addressed to Maryam Rahimi; maryam.b.rahimi@gmail.com
}

Received 16 December 2014; Revised 12 March 2015; Accepted 12 March 2015

Academic Editor: Christoph F. Mecklenbräuker

Copyright (c) 2015 M. Rahimi and E. Foroozanfard. This is an open access article distributed under the Creative Commons Attribution License, which permits unrestricted use, distribution, and reproduction in any medium, provided the original work is properly cited.

\begin{abstract}
The investigation on the achieved data rate of the cellular system considering different grips of handsets at different frequencies using measurement results of the measurement campaign-which was carried out in the city of Aalborg-is presented in this paper. The achieved data rate of the multiple-input single-output (MISO) interference channel is investigated. A typical propagation environment using two BSs and four handsets, like smart phones, held by four to eight different users was designed and multipleinput multiple-output (MIMO) channels in different scenarios were measured. In this paper, two BSs and two handsets at each measurement time are considered. The impact of the different parameters like correlation, different grips of handsets, and different long term evolution (LTE) frequency bands on the achieved data rate is investigated for different measurements. It could be concluded that the variations in the values of data rate are weakly associated with the different grips of handsets but more correlated with different frequencies.
\end{abstract}

\section{Introduction}

Cellular MIMO systems attract the interest of researchers due to providing continuous services to mobile users, enhancing system performance, and gaining higher data rate at the receiver [1]. The main limitation in achieving the promised capacity in the network MIMO is caused by interference from their own base stations or mobile terminals $[2,3]$. Due to that limitation research on interference channels become a vital issue $[4,5]$.

To improve the capacity and system performance of cellular networks, beamforming for downlink multicell MIMO systems has been proposed as one of the most promising techniques to solve the interference problem [6-9]. Linear transmission techniques because of their simplicity in comparison with nonlinear ones have attracted the most interest from researchers [10-13]. Among different proposed algorithms, the VSINR algorithm has been chosen in this work to analyze the measured data due to the simplicity and high performance of the algorithm. The performance of the algorithm is validated with the real propagation environment, while measured channels are used. VSINR maximization algorithm proposed by [14] adapts the weight coefficients to the original VSINR proposed scheme [15] to achieve a desired weighted sum-rate maximizing point. The updated VSINR algorithm proposed by [14] is used in this work.

After implementing the proper algorithm to analyze the measured channels, different parameters and their effects on the achieved data rate are investigated in this work. The measurement was done at two different frequencies (776 and $2300 \mathrm{MHz}$ ) simultaneously with regard to the LTE standard. Different scenarios with respect to the low and high band were investigated to compare the performance of the system. This was done to find an answer to this important question about which frequency promises more data rate in the measured channels, which is a novel work in this field to the knowledge of the author.

On the other hand, many different scenarios have been considered in the measurement campaign. One of them is the investigation on the influence of different grips on the achieved data rate. Eight different users held the handsets during measurements. The users were instructed to hold the handsets with the predefined grips and walk forward and backward inside a square which is drawn on the floor. The 
effect of different grips in MIMO case has been studied for previous measurement campaign $[16,17]$. In this work more grips added to the measurement scenarios and the effect of them on the performance of the algorithm will be discussed in the cellular network systems.

The last parameter which is investigated in this work is cross correlation between different links from the base stations to the users. The designed system works as each BS serves its user, so signals received by the second user are assumed as the interference. To investigate whether cross correlation between channels has any effect on the achieved data rate, the cross correlation between all links from each BS to each user is computed, which is called correlation for desired links, the same procedure is applied for the links from each BS to the second user and the correlation is called interference correlation. The effect of these values on the behavior of the algorithm will be shown in this work. This investigation is a new look through the effect of the correlation on the data rate, although there have been some studies on the effect of the correlation on the capacity of MIMO in previous measurement campaigns [18-20]. It should be noted that the effect of the other parameters such as fading, branch power ratio, different handset efficiencies, and different distances to the base stations is taken into account automatically, when the real environment is considered. These effects degrade the performance of the algorithm from simulation to the measurement. But the main focus of this paper is the investigation of the different grips, correlation, and different frequencies.

All results from measured data will be shown statistically utilizing a great amount of data. The median and variance of different sets of data have been plotted to have a fair comparison between different scenarios. Also from now on, we use the word "capacity" instead of achieved data rate for its simplicity.

\section{Measurement Setup}

2.1. Scenario and Setup. The measurements used in this work are described in [21] and a brief summary is given below.

The measurement campaign is situated in an urban macrocellular environment in the center of the city of Aalborg, Denmark. The measurement scenario has been designed to consider the cooperation between BSs. To fulfill this requirement, two base stations were installed at two different positions. The compromise of both high capacity and coverage has been provided in the setup because BS1 was assumed to provide high capacity channels, so it was located at $13 \mathrm{~m}$ height and $150 \mathrm{~m}$ away from the measurement area. BS2 was located at $500 \mathrm{~m}$ from the measurement building but at the height of $60 \mathrm{~m}$ to support more coverage in the cellular system. Each BS has four transmit antennas, two at low band and two at high band frequency based on LTE standards. Figure 1 shows the locations of the base stations. Two bands were measured simultaneously. An effective sounding bandwidth of about $5 \mathrm{MHz}$ was used at the center frequency of $776 \mathrm{MHz}$. This band is subsequently referred to as the low band (LB). The high band (HB) was centered at $2300 \mathrm{MHz}$ where an effective sounding bandwidth of about $100 \mathrm{MHz}$ was used. The two bands were chosen to resemble the LTE bands in the $700-800 \mathrm{MHz}$ and $2.3-2.6 \mathrm{GHz}$ ranges, respectively. In
TABLE 1: Handsets and antennas overview.

\begin{tabular}{lcc}
\hline Handset & Antenna number & Antenna location \\
\hline H1 & 1 & Top-center \\
PDA & 2 & Bottom-center \\
\hline H2 & 1 & Top-right \\
PDA & 2 & Bottom-right \\
\hline H5 & 1 & Top-right \\
PDA & 2 & Top-left \\
\hline H6 & 1 & Top-right \\
PDA & 2 & Top-center \\
\hline
\end{tabular}

practice, both the center frequencies and the bandwidths are compromises given the available equipment and unused frequency spectrum, resulting in the unequal bandwidths. In this work we chose both frequencies to investigate the performance of the algorithm at different frequencies.

The measurement setup is very close to the transmission method, which will be applied in practice, since transmission has been measured simultaneously from both base stations to four different users.

The measurement room is located on the third floor of the Aalborg university (AAU) building in the city center. The room has no windows toward any BS, which means line of sight (LOS) was blocked. In the room, four $1 \mathrm{~m}$ by $1 \mathrm{~m}$ squares were drawn on the floor. Users moved back and forth inside squares during the measurement time. This pattern of movement is called local average (LA). The measurement was done with different handsets and users, which will be described in the next part.

2.2. Handsets and Users. Ten different handsets were designed and measured in this campaign. In this work we focus on four of them $(\mathrm{H} 1, \mathrm{H} 2, \mathrm{H} 5$, and $\mathrm{H} 6)$. Table 1 gives an overview of the antenna types and locations of the antennas at different handsets for reference. All handsets have a size of $59 \times 11 \mathrm{~mm}$. All antenna types are mono and all handsets have antennas at low and high band. This group of handsets was chosen because they have similar types of antennas. This selection is done to avoid any unwanted differences in the results, which could be caused by the differences in the type of antennas. Each handset has two antennas in LB and HB. Antennas were located in different positions in different handsets.

Each handset was connected to the sounder via optical fiber instead of coaxial cables to avoid the effect of changes in the electromagnetic properties on small devices. This solution helps to measure the handsets in a more natural and realistic way. The optical unit was designed to be small enough to fit into a typical handset [19].

In addition, each handset was covered in a plastic frame, which is close to the material of mobile phones for a more realistic scenario and avoiding users to touch printed circuit boards (PCB) directly. More details about the handsets could be found in [21].

Five different grips were used in this measurement. In each case, the users placed their fingers in predefined 


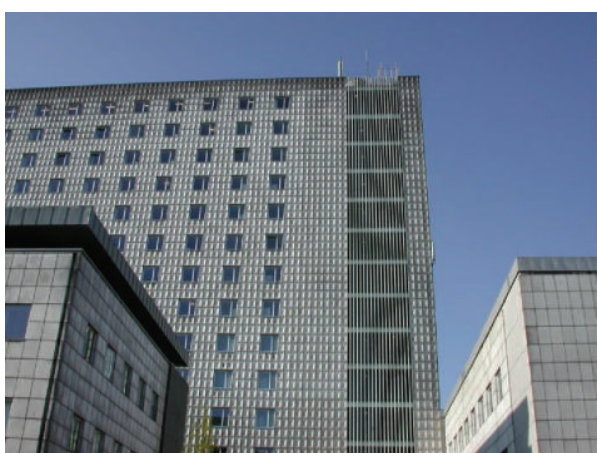

(a)

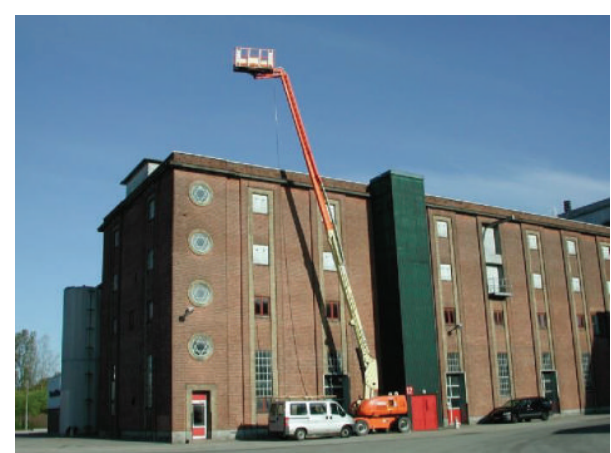

(b)

FIGURE 1: Positions of base stations (a) BS2, (b) BS1.

markings on the handsets and held the handset in front of the body at an angle of about $45^{\circ}$. Five different grips of handsets are considered: portrait mode right hand only (phr); portrait mode two hands (pth); landscape mode, right tilt, right hand only (lphr); landscape mode, right tilt, left hand only (lrhl); landscape mode, right tilt, two hands (lrth). The grips are shown in Figure 2. Moreover, eight different users were involved in this measurement to investigate the effects of different bodies on the receive antennas. Each handset was tested by different users and different grips. Different grips were studied to measure each handset. This involved users touching antennas or holding the handset in a certain way, so that their fingers were far from the receive antennas. In this work we included the effect of different grips on the performance of the system.

2.3. Investigated Scenario. Two BSs and two handsets at each measurement time were considered to compute the capacity. Two antennas at each base station and one single antenna per each handset were selected, which all were working in narrow band at $2.3 \mathrm{GHz}$ and $776 \mathrm{MHz}$ frequency. Figure 3 illustrates the investigated scenario in this work. The BSs shared the channel knowledge and they canceled the interference from the other BS.

Figure 4 illustrates the position and potential orientation of movements of the users. Each small square has the dimension of $1 \times 1 \mathrm{~m}^{2}$. Users moved randomly back and forth in the squares during each measurement.

\section{Data Analysis}

In this section different steps for analyzing the measured data and the normalization method will be introduced.

3.1. Channel Normalization. There are different ways of normalizing the channel for different scenarios with respect to the different parameters, which we want to investigate in the capacity.

In this work, the difference between power levels from two base stations should be preserved. So the received mean power by the reference handset (H6) in all free space

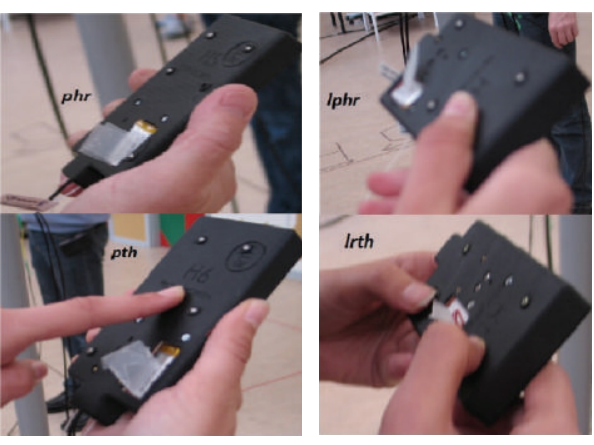

FIGURE 2: Different grips of handsets.

measurements from one base station is computed, in this normalization, we can see the influence of the following:

(1) branch power ratio,

(2) correlation,

(3) different power levels from different base stations,

(4) effect of different handsets,

(5) fading information.

The equation for this normalization is as follows:

$$
h_{n, m}^{\text {norm }}(g)=\frac{h_{n, m}(g)}{\sqrt{(1 / N M G) \sum_{n, m, g} h_{n, m}^{\text {ref }}(g)^{2}}},
$$

where $M=2$ is the number of transmit antennas, $N=1$ is the number of receive antennas, and $G=1200$ is different samples in each measurement, when $g$ can be interpreted as the time index and $h_{n, m}$ are the channel coefficients from $n$th receive antenna to the $m$ th transmit antenna and $h_{n, m}^{\text {ref }}(g)$ are the channels for the reference handset in free space.

3.2. Correlation. To investigate the effect of correlation on the capacity, correlation coefficients between links from the measurement data are computed. The correlation is computed between desired links and interference links separately. Desired links mean direct links from each BS towards its user, 


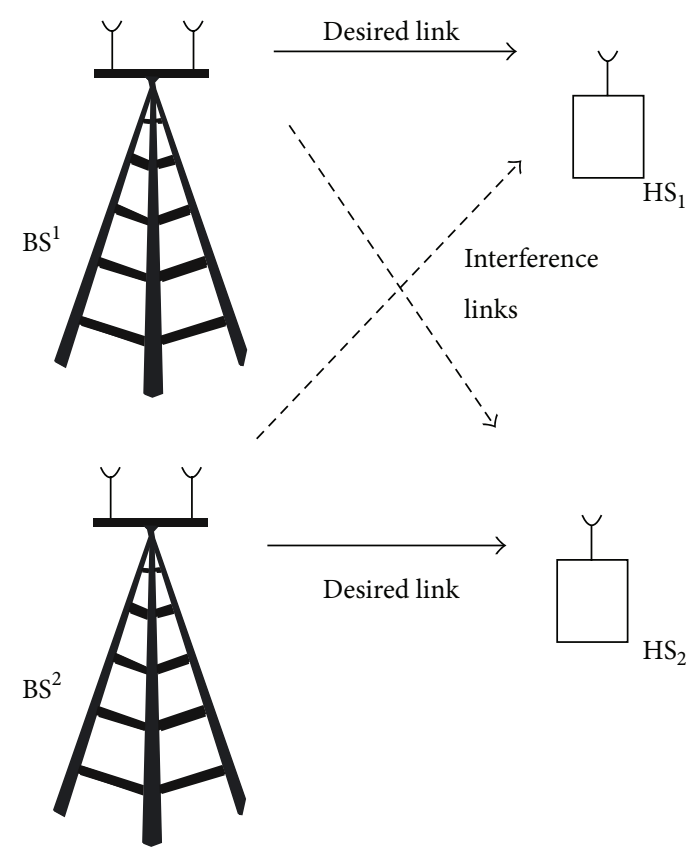

FIGURE 3: Investigated scenario.



FIgURE 4: Measurement room.

like BS1 serves user 1. Interference links are channels from each BS to the other user (BS1 to user 2 and vice versa). The links are shown in Figure 3. The correlation coefficients are calculated using the Pearson correlation coefficient equation as defined in [22].

3.3. VSINR Algorithm. The VSINR algorithm works for $K$ users and $K$ base stations scenario. But in this work, two users and two BSs are assumed for the simulation, with two transmit and one receive antenna for each. In the VSINR algorithm, nonweighted coefficients are assumed and the average WSR was computed. The results presented in [14] included weighted coefficients. It means that the VSINR algorithm used in this work was implemented for the reduced version of the original one and could have even better performance if we use the same assumptions as the reference. For example, using weight coefficients of [3 1 1] leads to achieve data rate at SNR $=15 \mathrm{~dB}$ around $19 \mathrm{bits} / \mathrm{sec} / \mathrm{Hz}$ [14], but it is $9 \mathrm{bits} / \mathrm{sec} / \mathrm{Hz}$ in

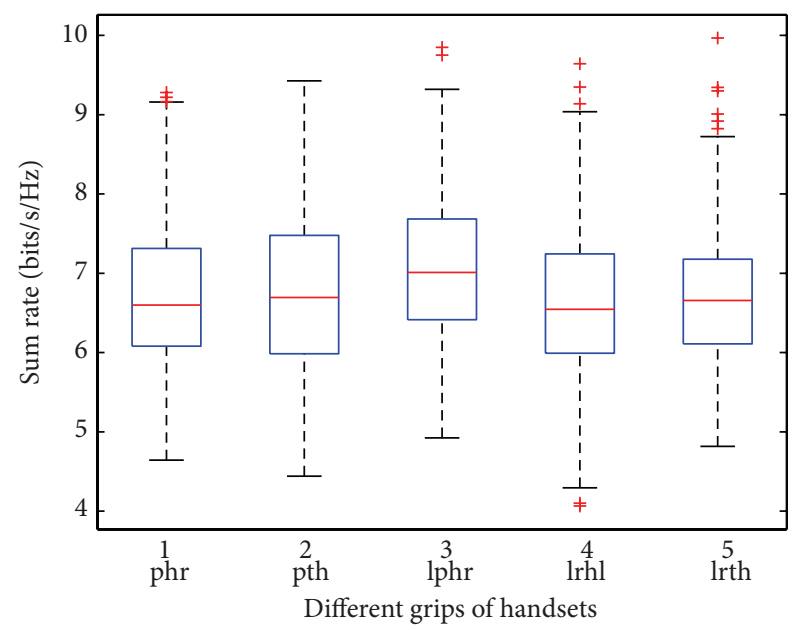

FIGURE 5: Capacity of different grips at high band.

our assumption. The reason of having this assumption is for investigating the lower bound of the VSINR algorithm with the measured data. It is clear that having the same results as it is shown in [14] is straightforward.

\section{Results and Discussion}

4.1. Capacity of Different Grips of the Measured Data Using VSINR Algorithm. In all measurements the SNR is fixed to $15 \mathrm{~dB}$. The achieved sum rate or capacity of different grips of HSs is computed and presented in this section using VSINR algorithm at low and high bands. The results show the sensitivity of the algorithm to the different grips and the investigation helps to understand the performance of the algorithm with the measured channels, as well as the behavior of the algorithm at different frequencies.

Figure 5 shows the capacity of different grips at high band frequency. Each box plot in the graph is plotted over a range between 403 and 420 different values based on successful measurements for each grip. As it is shown, the results have almost the same variations. The median of lphr is the highest among all of them and phr and lrth gained the lowest values. The difference between the highest and the lowest value is around $0.4 \mathrm{bits} / \mathrm{sec} / \mathrm{Hz}$.

Figure 6 presents the same results but at low band frequency. The same trends could be found in the capacities of different grips. The lphr and lrth have the highest median value of $8.6 \mathrm{bits} / \mathrm{sec} / \mathrm{Hz}$ and the rest gained the lowest value of $8.3 \mathrm{bits} / \mathrm{sec} / \mathrm{Hz}$. It shows different frequencies do not have significant effect on the capacity of different grips. But in general the mean of the medians at low frequency is around $1.7 \mathrm{bits} / \mathrm{sec} / \mathrm{Hz}$ higher than the mean of the medians at high frequency. More investigation on other parameters like correlation could explain these differences.

4.2. Effect of Correlation on the Capacity. The correlation is plotted for desired links and interference links as mentioned before at both frequencies. Based on our setup, each desired or interference link has two entries, which are channels from 


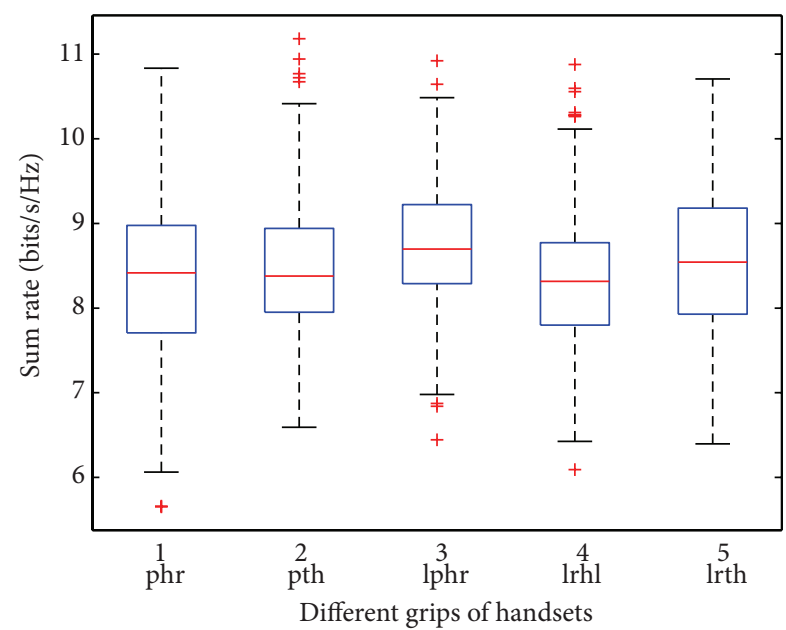

FIGURE 6: Capacity of different grips at low band.

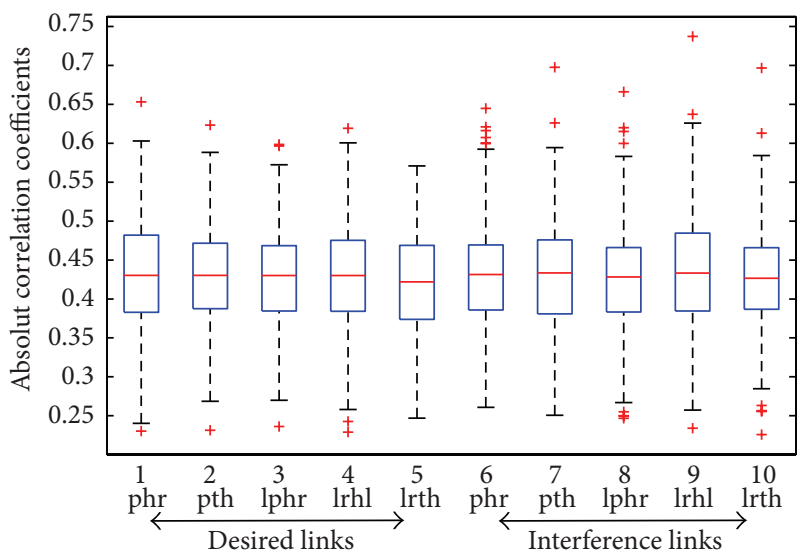

FIGURE 7: Correlation coefficients for different grips at high band.

each antenna at the user towards each antenna at each BS. The correlation coefficients between channels from each BS towards each HS are computed first. The mean of values has been calculated and assumed as the correlation for the desired link. The same procedure has been done for interference links, this time for the links from BS1 towards HS2 and vice versa.

Considering correlation for each of the grips, which are shown in Figures 7 and 8 for high and low band, respectively, there are no obvious differences in the median values. The results have good agreement with observations from Figures 5 and 6 , while there were not significant differences in the median values of the capacities in terms of different grips. However, by comparing low and high band, we can see low band has lower correlation around 0.1 than high band based on the mean of median values, although in total, channels are not very correlated. On the other hand, the absolute values of the correlations have a wide variation, which affect the capacity individually. To investigate the relation between correlation and capacity, the scatter plot is presented.

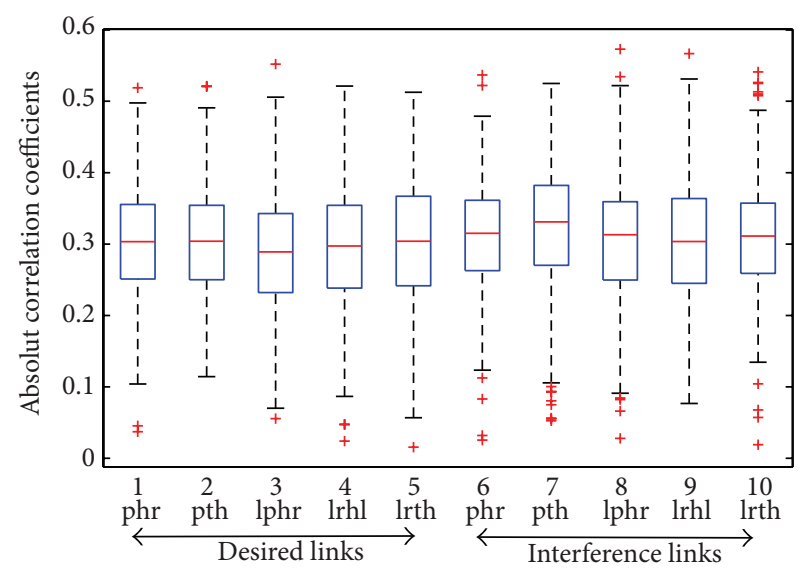

FIGURE 8: Correlation coefficients for different grips at low band.

Figures 9 and 10 show the scatter plots for correlation versus capacity for desired and interference links, respectively, in terms of different grips at high band. From the plots it is evident that desired links have wider distribution in correlation than in interference links. However, at both figures, the solid pattern of changes in the capacity with regard to the correlation cannot be found.

The same investigation is done for low band. Figures 11 and 12 show the results. The distribution of the correlation is the same for desired and interference links and again, strong links between changes in the correlation with regard to capacity could not be seen. Comparison of these four figures illustrates a wider range of correlation in LB than HB. As can be seen at LB, correlation varies from 0 to 0.5 with most aggregation around 0.4. At $\mathrm{HB}$, the variation is from 0.2 to 0.7 , with most of the values placed at 0.45 . To present a more solid conclusion, Figure 13 is presented.

Figure 13 shows the scatter plots for correlation versus capacity for HB and LB in desired and interference links. The effect of each grip is not shown in these results. A pattern can be seen that capacity decreases dramatically with an increase in correlation. Regardless of desired or interference links, low bands (yellow and blue stars) have lower correlation and higher capacity, which can explain the differences of capacities in Figures 5 and 6.

4.3. Repeatability. The measure of comparison in this work is cooperative capacity between two BSs and two HSs at each time. In order to check the reliability of the measurement, it is important to investigate the repeatability of the measurements in the same combination. In this measurement the repeated data is available for free space, which means there is no user to hold the handsets. $\mathrm{H} 6$ and $\mathrm{H} 5$ are chosen to check the capacity. In 18 different measurements, around $72 \%$ of them have a difference to the mean value of less than $0.1 \mathrm{bits} / \mathrm{sec} / \mathrm{Hz}$. And $5 \%$ of them considered as error in the measurement, because they have a difference to the mean value of more than $1 \mathrm{bits} / \mathrm{sec} / \mathrm{Hz}$. However, the errors were attributed to noise in the measurement or some changes in the measurement environment. In general, we can conclude 


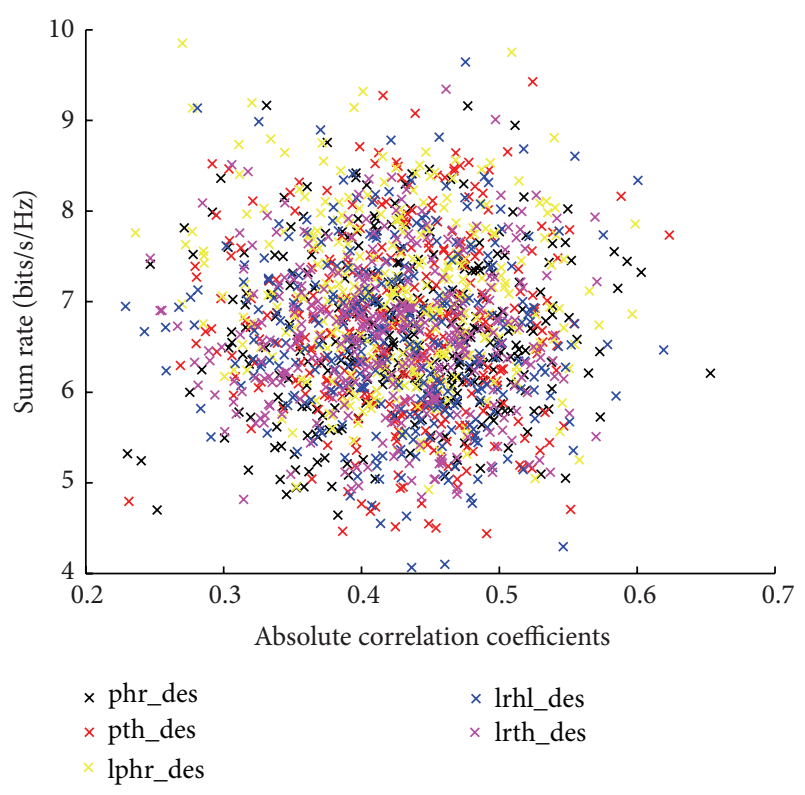

FIGURE 9: Scatter plot: capacity versus correlation for desired links at high band.

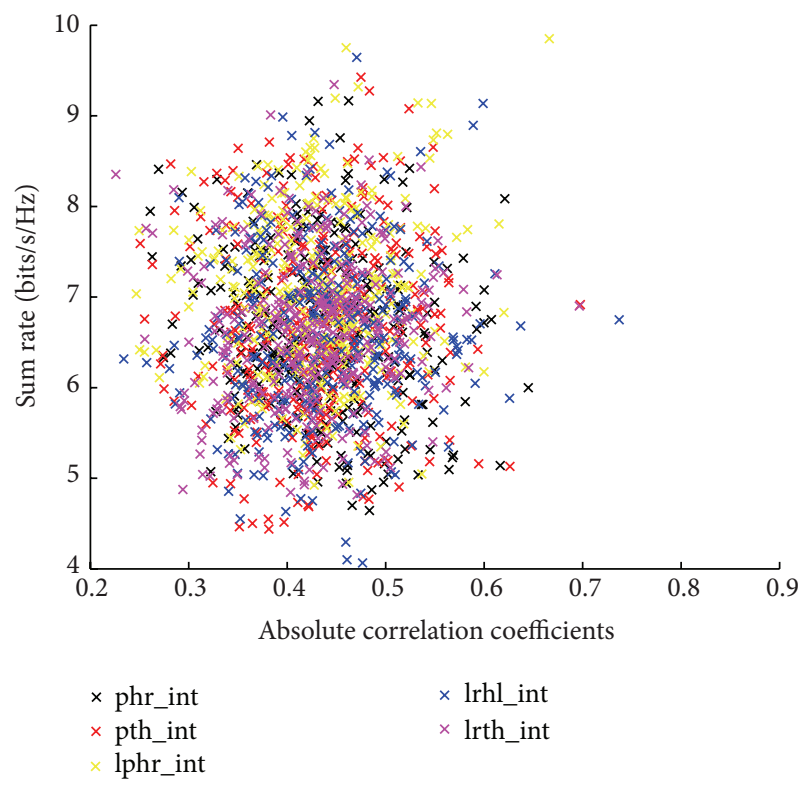

FIGURE 10: Scatter plot: capacity versus correlation for interference links at high band.

that the most cases are error-free and that the measurement was very accurate.

\section{Conclusion}

This work analyzed the results from a recent measurement campaign which was carried out in May 2011 in central Aalborg city. The main focus of the work was the capacity of the interference channels. To find the capacity, the VSINR algorithm was implemented in MATLAB using measured channels.

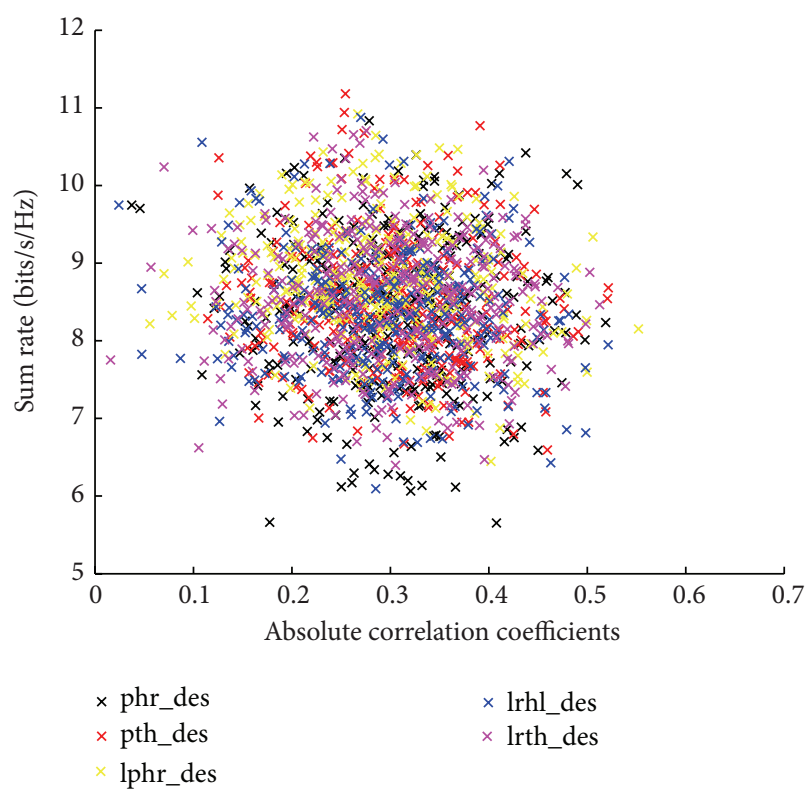

FIGURE 11: Scatter plot: capacity versus correlation for desired links at low band.

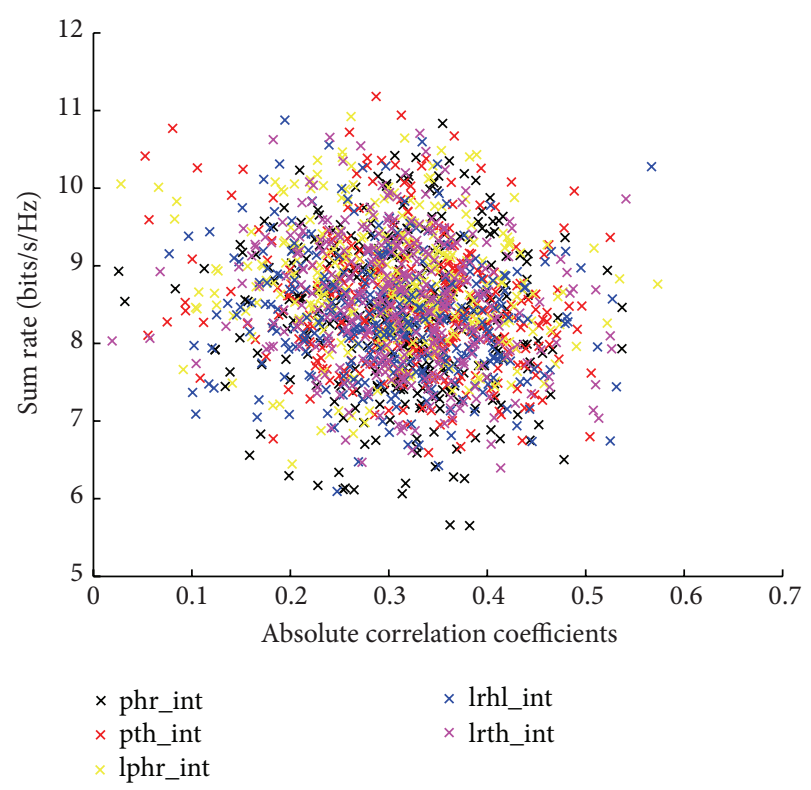

FIGURE 12: Scatter plot: capacity versus correlation for interference links at low band.

The behavior of the algorithm in different frequencies with measured channels was investigated in this work. The results show the following.

(1) The highest capacity was achieved in low band (the median is $8.6 \mathrm{bits} / \mathrm{sec} / \mathrm{Hz}$ ).

(2) The algorithm was not very sensitive to the different grips of handsets.

(3) Overall channels are more correlated at high band, although the difference between low and high band 


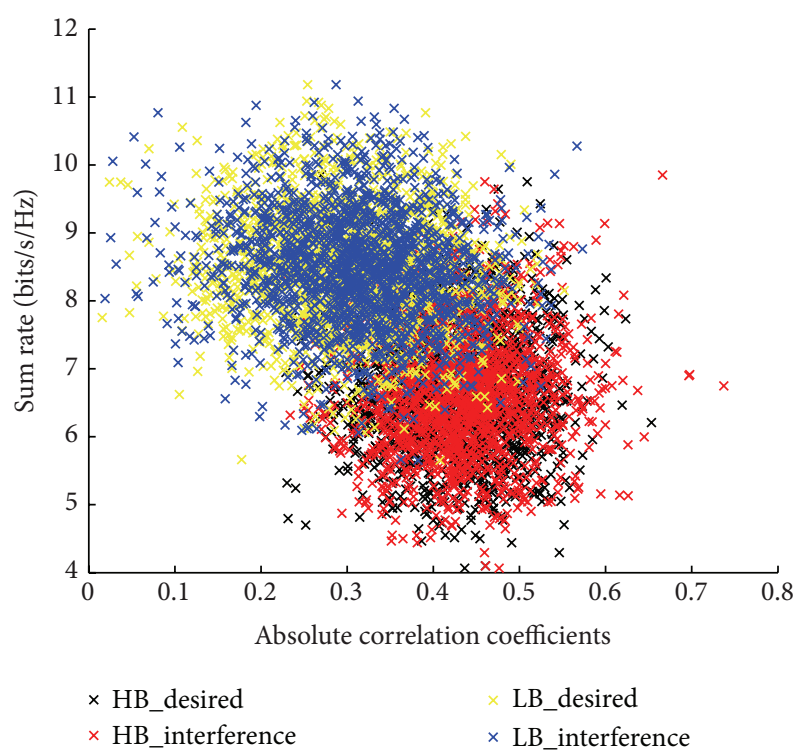

FIgURE 13: Scatter plot: capacity versus correlation for desired and interference links at low and high band.

is not very significant (around 0.1). These differences cause higher capacity at low band.

It should be noted that, as long as signals at low band have better penetration (meaning they pass through objects such as walls with less attenuation), they can lead to a higher data rate in inside environments, where our measurement was done. In [23] same analysis has been studied. The penetration loss is computed for high and low band for indoor scenario, which is very close to the presented scenario in this work. The results also confirm that low band signals have better penetration and they can pass through objects better than high band signals. The important highlight of this work is that working at low band leads to a higher data rate in addition to other advantages like better coverage, more penetration, and lower price to establish setup.

\section{Conflict of Interests}

The authors declare that there is no conflict of interests regarding the publication of this paper.

\section{References}

[1] S. Shamai Shitz, O. Somekh, and B. M. Zaidel, "Multi-cell communications: an information theoretic perspective," in Proceedings of the Joint Workshop on Communications and Coding (JWCC '04), Florence, Italy, October 2004.

[2] S. Catreux, P. F. Driessen, and L. J. Greenstein, "Attainable throughput of an interference-limited multiple-input multipleoutput (MIMO) cellular system," IEEE Transactions on Communications, vol. 49, no. 8, pp. 1307-1311, 2001.

[3] R. S. Blum, "MIMO capacity with interference," IEEE Journal on Selected Areas in Communications, vol. 21, no. 5, pp. 793-801, 2003.
[4] J. G. Andrews, W. Choi, and R. W. Heath Jr., "Overcoming interference in spatial multiplexing MIMO cellular networks," IEEE Wireless Communications, vol. 14, no. 6, pp. 95-104, 2007.

[5] H. Sung, S.-H. Park, K.-J. Lee, and I. Lee, "Linear precoder designs for K-user interference channels," IEEE Transactions on Wireless Communications, vol. 9, no. 1, pp. 291-301, 2010.

[6] A. Tolli, M. Codreanu, and M. Juntti, "Cooperative MIMOOFDM cellular system with soft handover between distributed base station antennas," IEEE Transactions on Wireless Communications, vol. 7, no. 4, pp. 1428-1440, 2008.

[7] H. Zhang, N. B. Mehta, A. F. Molisch, J. Zhang, and H. Dai, "Asynchronous interference mitigation in cooperative base station systems," IEEE Transactions on Wireless Communications, vol. 7, no. 1, pp. 155-165, 2008.

[8] Y. Hadisusanto, L. Thiele, and V. Jungnickel, "Distributed base station cooperation via block-diagonalization and dualdecomposition," in Proceedings of the IEEE Global Telecommunications Conference (GLOBECOM '08), pp. 3539-3543, December 2008.

[9] A. Ligata, H. Gacanin, and T. Javornik, "On performance of MIMO-OFDM/TDM using MMSE-FDE with nonlinear HPA in a multipath fading channel," IEICE Transactions on Communications, vol. 97, no. 9, pp. 1947-1957, 2014.

[10] M. Schubert and H. Boche, "Iterative multiuser uplink and downlink beamforming under SINR constraints," IEEE Transactions on Signal Processing, vol. 53, no. 7, pp. 2324-2334, 2005.

[11] W. Yu and T. Lan, "Transmitter optimization for the multiantenna downlink with per-antenna power constraints," IEEE Transactions on Signal Processing, vol. 55, no. 6, pp. 2646-2660, 2007.

[12] A. Wiesel, Y. C. Eldar, and S. Shamai, "Linear precoding via conic optimization for fixed MIMO receivers," IEEE Transactions on Signal Processing, vol. 54, no. 1, pp. 161-176, 2006.

[13] T. Yoo and A. Goldsmith, "On the optimality of multiantenna broadcast scheduling using zero-forcing beamforming," IEEE Journal on Selected Areas in Communications, vol. 24, no. 3, pp. 528-541, 2006.

[14] S.-H. Park, H. Park, and I. Lee, "Beamforming design based on virtual SINR maximization for interference networks," in Proceedings of the IEEE International Conference on Communications (ICC '11), pp. 1-5, June 2011.

[15] R. Zakhour and D. Gesbert, "Distributed multicell-MISO precoding using the layered virtual SINR framework," IEEE Transactions on Wireless Communications, vol. 9, no. 8, pp. 2444-2448, 2010.

[16] J. Ø. Nielsen, B. Yanakiev, I. B. Bonev et al., "User influence on the mean effective gain for data mode operation of mobile handsets," in Proceedings of the 6th European Conference on Antennas and Propagation (EuCAP '12), pp. 2759-2763, March 2012.

[17] J. Ø. Nielsen, B. Yanakiev, S. C. Del Barrio, and G. F. Pedersen, "Channel statistics for MIMO handsets in data mode," in Proceedings of the 8th European Conference on Antennas and Propagation (EuCAP '14), pp. 2818-2822, The Hague, the Netherlands, April 2014.

[18] S.-H. Lee and S.-Y. Chung, "Effect of channel correlation on the capacity scaling in wireless networks," in Proceedings of the IEEE International Symposium on Information Theory (ISIT '08), pp. 1128-1132, IEEE, Toronto, Canada, July 2008.

[19] B. Yanakiev, J. Ø. Nielsen, and G. F. Pedersen, "On small antenna measurements in a realistic MIMO scenario," in Proceedings 
of the 4th European Conference on Antennas and Propagation (EuCAP '10), pp. 1-5, IEEE, Barcelona, Spain, April 2010.

[20] B. Yanakiev, J. Ø. Nielsen, M. Christensen, and G. F. Pedersen, "Correlation measurements on small mobile devices," in Proceedings of the 6th European Conference on Antennas and Propagation (EuCAP '12), pp. 382-385, March 2012.

[21] J. O. Nielsen, B. Yanakiev, S. C. Del Barrio, and G. F. Pedersen, "On antenna design objectives and the channel capacity of MIMO handsets," IEEE Transactions on Antennas and Propagation, vol. 62, no. 6, pp. 3232-3241, 2014.

[22] Mathworks May, Matlab, 2010, http://www.math-works.com/ help/toolbox/stats/corr.html.

[23] H. Elgannas and I. Kostanic, "Outdoor-to-indoor propagation characteristics of $850 \mathrm{MHz}$ and $1900 \mathrm{MHz}$ bands in macro cellular environments," in Proceedings of the World Congress on Engineering and Computer Science (WCECS '14), October 2014. 



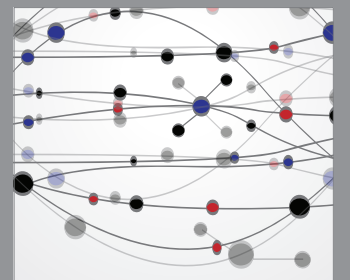

The Scientific World Journal
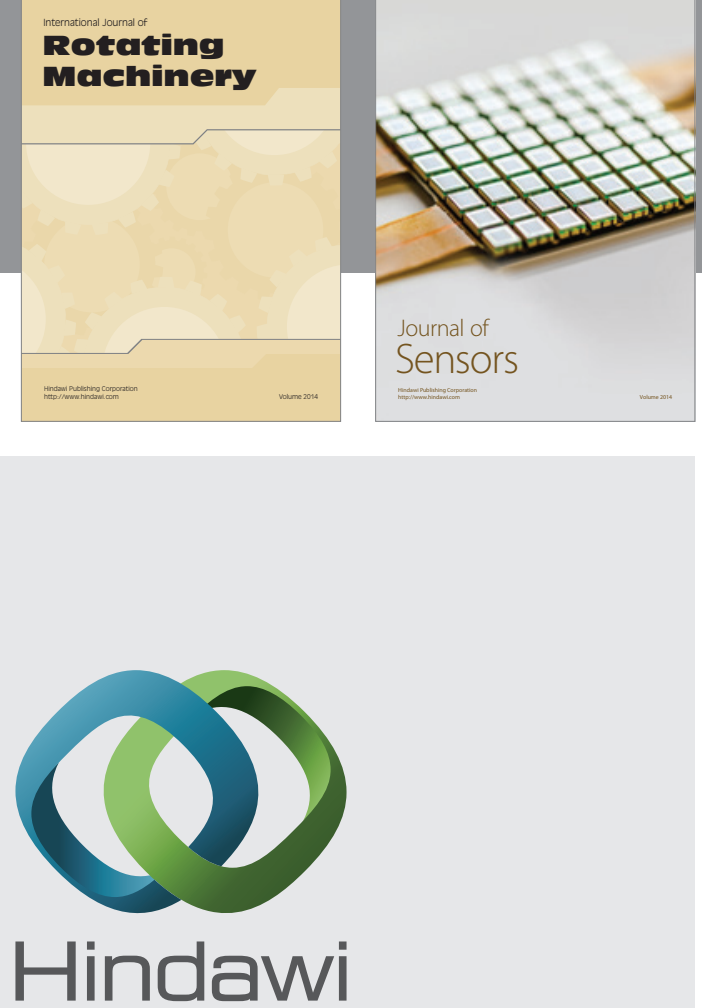

Submit your manuscripts at http://www.hindawi.com
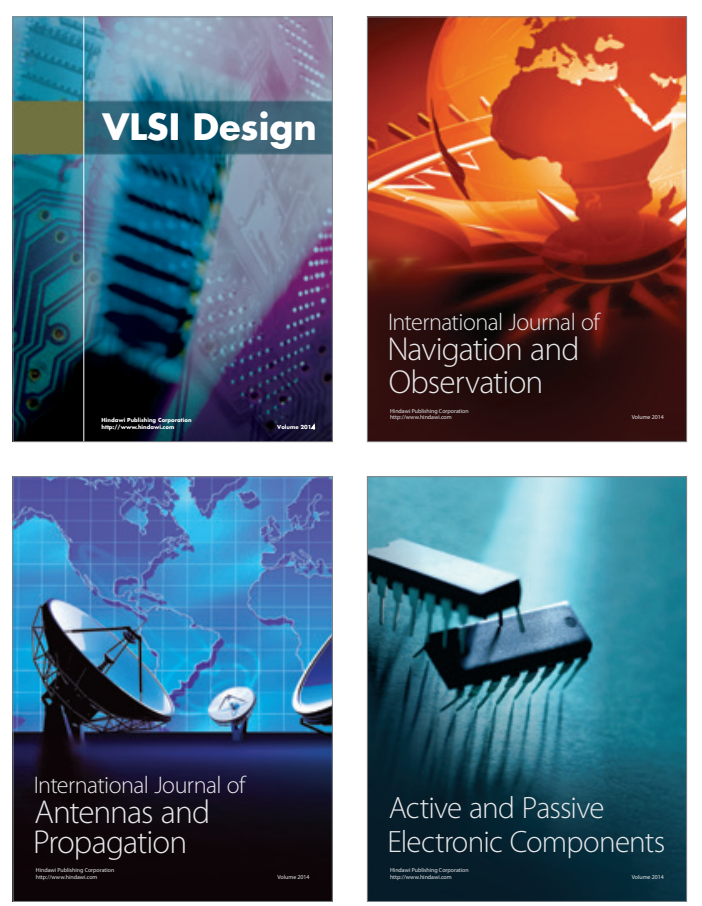
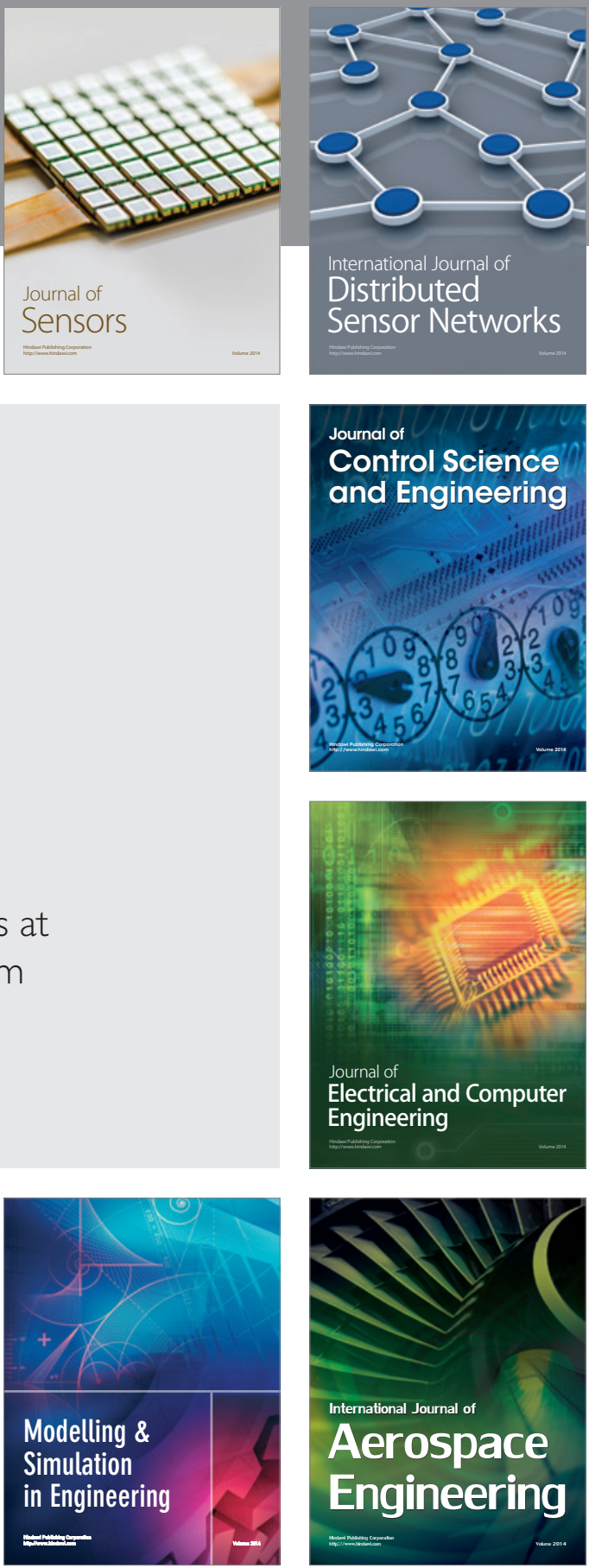

Journal of

Control Science

and Engineering
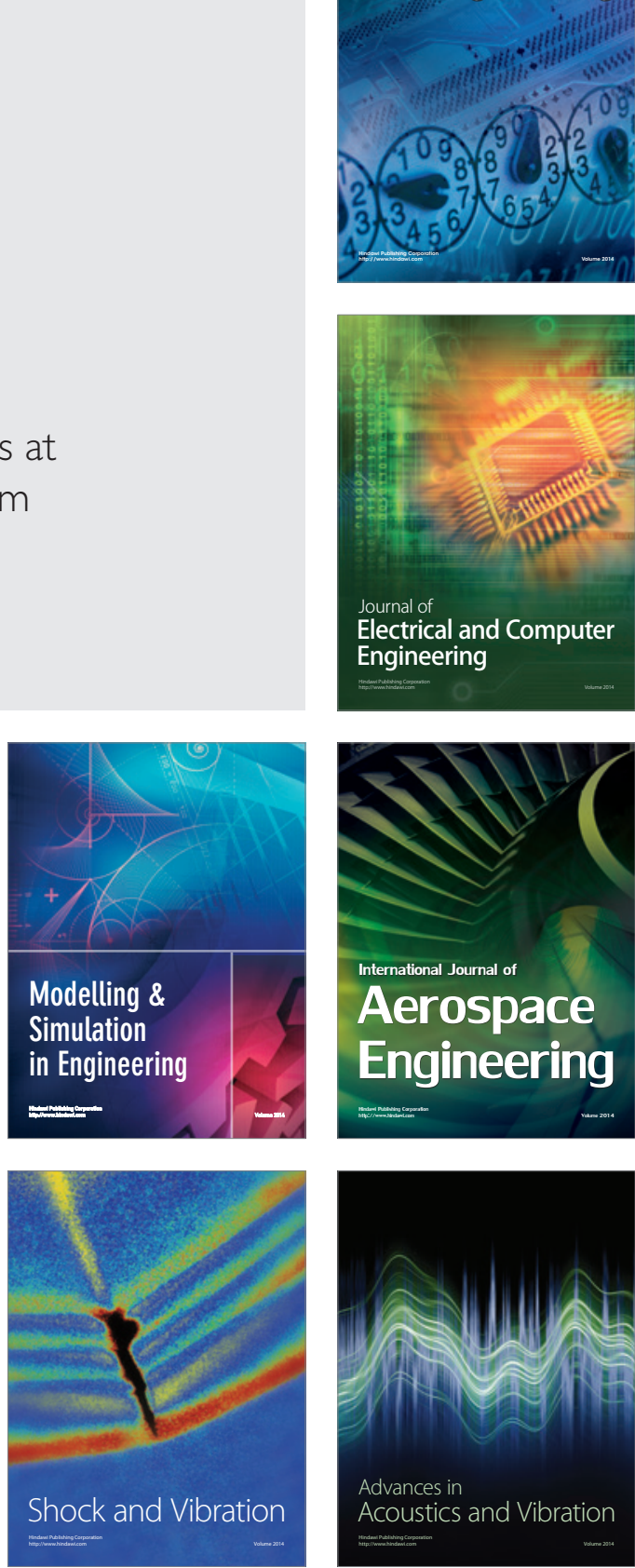